\section{A FIB Micro-Sampling Technique for Three-Dimensional Characterization of a Site-Specific Defect}

Toshie Yaguchi ${ }^{1}$, Yasushi Kuroda ${ }^{1}$, Mitsuru Konno ${ }^{1}$, Takeo Kamino ${ }^{1}$, Tsuyohsi Ohnishi ${ }^{2}$, Takahito Hashimoto $^{2}$, Kaoru Umemura ${ }^{2}$, Kyoichiro Asayama ${ }^{3}$

${ }^{1}$ Hitachi Science Systems, Ltd., Japan

${ }^{2}$ Hitachi High-Technologies Corporation, Japan

${ }^{3}$ Renesas Technology Corp., Japan

In characterization or failure analysis of new materials and semiconductor devices, the requirements for three dimensional observation and analysis are rapidly increasing. We discuss techniques for specimen preparation, three-dimensional observation, and elemental analysis of semiconductor devices that we developed using a system consisting of a dedicated focused ion beam (FIB) instrument and a scanning transmission electron microscope (STEM). The system utilizes a FIB-STEM compatible specimen holder with a specially designed rotation mechanism, which allows $360^{\circ}$ rotation of a specimen on a conical stage (needle stub) around the ion beam axis of the FIB system and $360^{\circ}$ rotation perpendicular to the electron beam in the STEM1,2. A piece of sample (micro sample) is extracted from a specific-site by the micro-sampling technique 3,4 and mounted on the needle stub. Instruments used in the study are the Hitachi FB-2100 FIB system with a micro-sampling attachment and the HD-2300 field emission 200kV STEM.
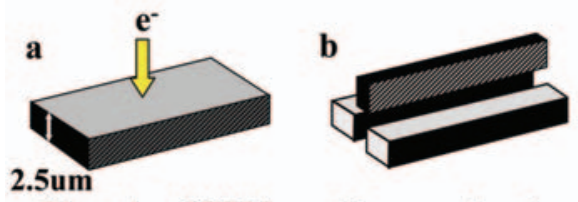

Plan-view STEM observation

\section{Cross-sectional} Sample preparation

c

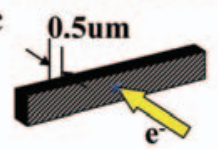

Cross-sectional STEM observation

Figure 1. Schematic outline of plan and cross-sectional view TEM specimen preparation from the same site and respective observations.

\section{Plan-view, cross-sectional and plan-view observations} of an SRAM device

The micro-sampling technique allows cross-sectional and plan-view STEM sample preparation from the same initial material at a pre-specified location. Figure 1 shows an outline of the plan for cross-sectional view, STEM specimen preparation from the specified site and subsequent observations. First, a plan-view micro-sample with a thickness of 2.5 micron is prepared in the FIB system and is observed with the 200kV FE-STEM (a).
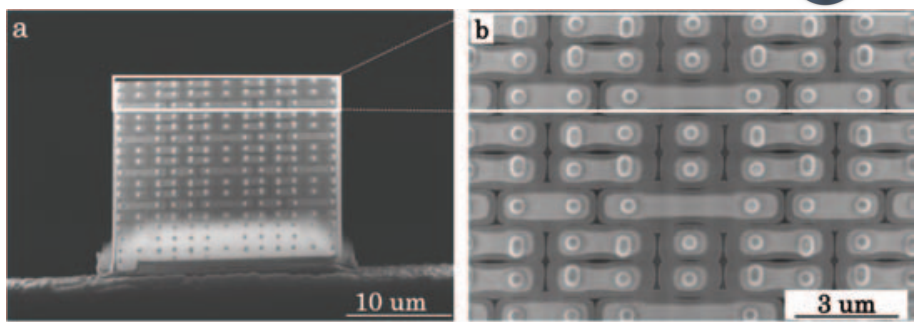

Figure 2. DF-STEM images of a plan-view sample observed at the accelerating voltage of $200 \mathrm{KeV}$. a.) The entire micro-sample, b.) enlarged image.

Since STEM imaging has less chromatic aberration than TEM imaging at the same $\mathrm{kV}$, the image quality in the STEM does not suffer degradation, even with a 2.5 micron thick sample. Next, a 0.5 micron thick cross-sectional sample is prepared from the plan-view sample and analyzed in the STEM. Finally, a precisely located, thin, plan-view sample is extracted from the cross-sectional sample using the patented micro-sampling technique (d). The plan-view STEM image is observed and the sample may now be fully characterized for defects utilizing all of the analytical accessories the instrument is capable of supporting.

The method described above was applied to characterize an inter-level via (or plug) of an SRAM cell in a microcomputer chip. To begin with, a micro-sample of about 2.5 micron thick was prepared for plan-view STEM observation. Figure 2 shows the dark-field (DF) STEM images of the plan-view sample (a) and its magnified image (b). The position of the plugs and wiring can be clearly observed. Next the upper part of the specimen, the square masked area, is cut out for cross-sectional sample observation. The cross-sectional DF-STEM image of the sample with a thickness of 0.5 micron is shown in Fig.3. Along both sides of the plugs we see dark contrast areas that are openings or gaps.

Subsequently, the sample is thinned to a final thickness of approximately 0.1 micron. Figure 4 a shows a plan-view DFSTEM image corresponding to the area observed in the crosssectional STEM image. Figures $4 \mathrm{~b}$ and $\mathrm{c}$ are enlarged images of the left and center plugs, respectively. The plugs are fringed with the bright contrast of a Ti/TiN barrier metal layer. There are differences in shape and peripheral structure of both plugs. In Fig.

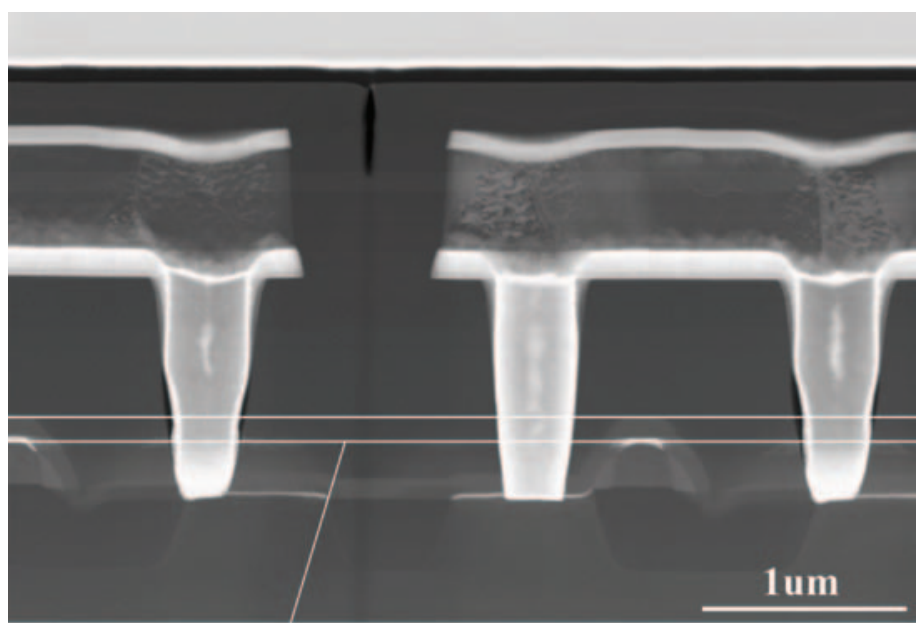

Area for plan-view sample

Figure 3. Cross-sectional DF-STEM image of an SRAM device specimen with a thickness of 0.5 microns, observed at $200 \mathrm{KeV}$. 
TGSTechnologies

TEM Sample Holder/Specimen Holder Service and Repair

\section{A NEW ALTERNATIVE TO YOUR TEM SPECIMEN HOLDER SERVICE AND REPAIR NEEDS.}

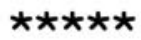

TGS Technologies Provides High Quality TEM Specimen Holder Service With 20 Years Experience. In addition we offer reasonable hourly service rates and more personalized service for you.

\section{Contact Us Today:}

TGS Technologies

Phone: 724.453.3865

E-mail: tom@tgstechnologies.net

Web: http://www.tgstechnologies.net
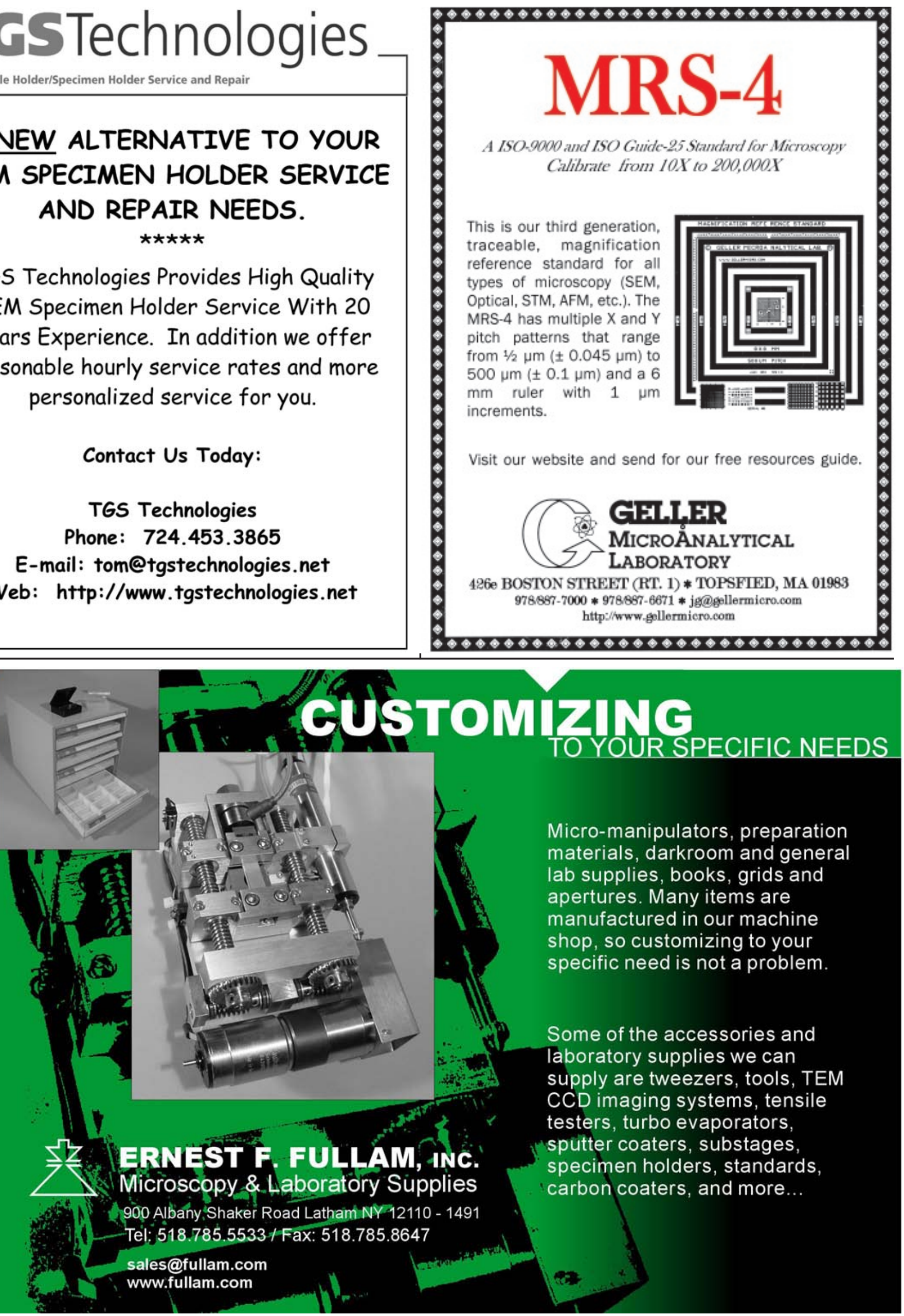

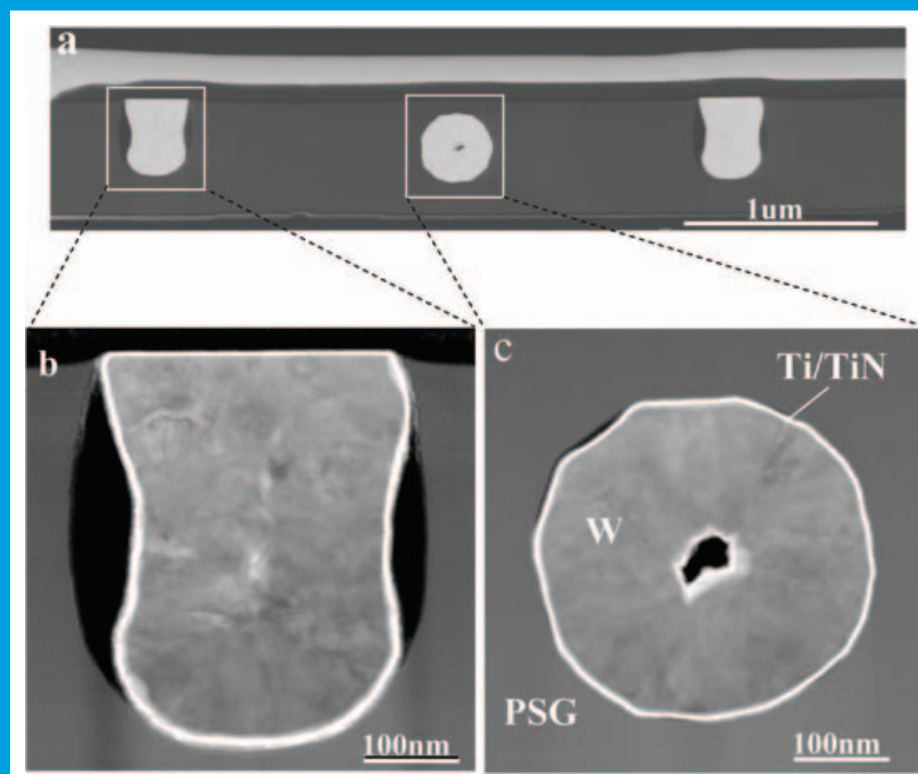

Figure 4. Plan-view DF-STEM images observed at $200 \mathrm{KeV}$ : a.) DF-

STEM image corresponding to the area observed in the figure 3 cross-section STEM;. b.) and c.) enlarged images of the left and center plugs.

$4 \mathrm{~b}$, there are openings between the phospho-silicate glass (PSG) insulating layer and the plug. It can be surmised that the PSG shrank when it was dried after the Ti/TiN layer was deposited.

\section{FIB micro-pillar sampling technique for 3D STEM obser - vation of DRAM Devices}

For this method, a piece of sample in the shape of a pillar (micro-pillar sample) is extracted at a pre-specified site for characterization by the FIB micro-sampling method. Figure 5 shows the procedure for this method. The extracted micro-pillar metal probe

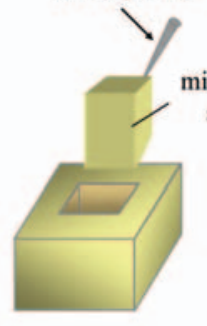

(a) Lifting out

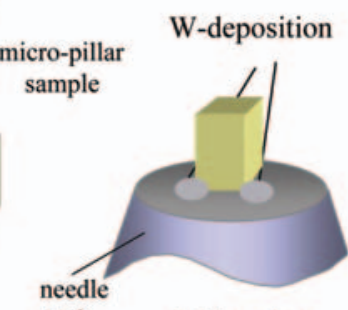

(b)Mounting

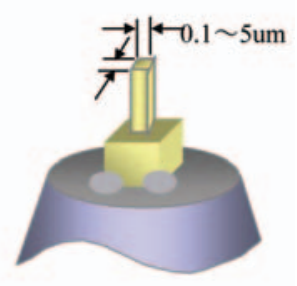

(c)FIB trimming
Figure 5. Schematic flow for the FIB micro-pillar specimen preparation technique.

sample (a) is transferred and mounted onto a needle stub (b). The sample is further shaped into a pillar of $\sim 0.1-5$ microns square and 10 to 15 microns long (c), and then transferred to the STEM for high angle annular dark field (HAADF) STEM, bright field STEM, and secondary electron microscopy (SEM) image observation at $200 \mathrm{kV}$ to obtain the three-dimensional and cross sectional structure of the specific-site. The geometry of the sample, and the needle stub, allows observation of the sample over a wide range of angles. In this manner, the specific site location is further refined for additional FIB milling as required to produce a final specimen. For a Si device, it takes less than 30 minutes to prepare a micro-pillar sample. A secondary electron (SE) image of a micro-pillar sample from a Si device mounted on a needle stub is shown in Fig. 6. The specific site can

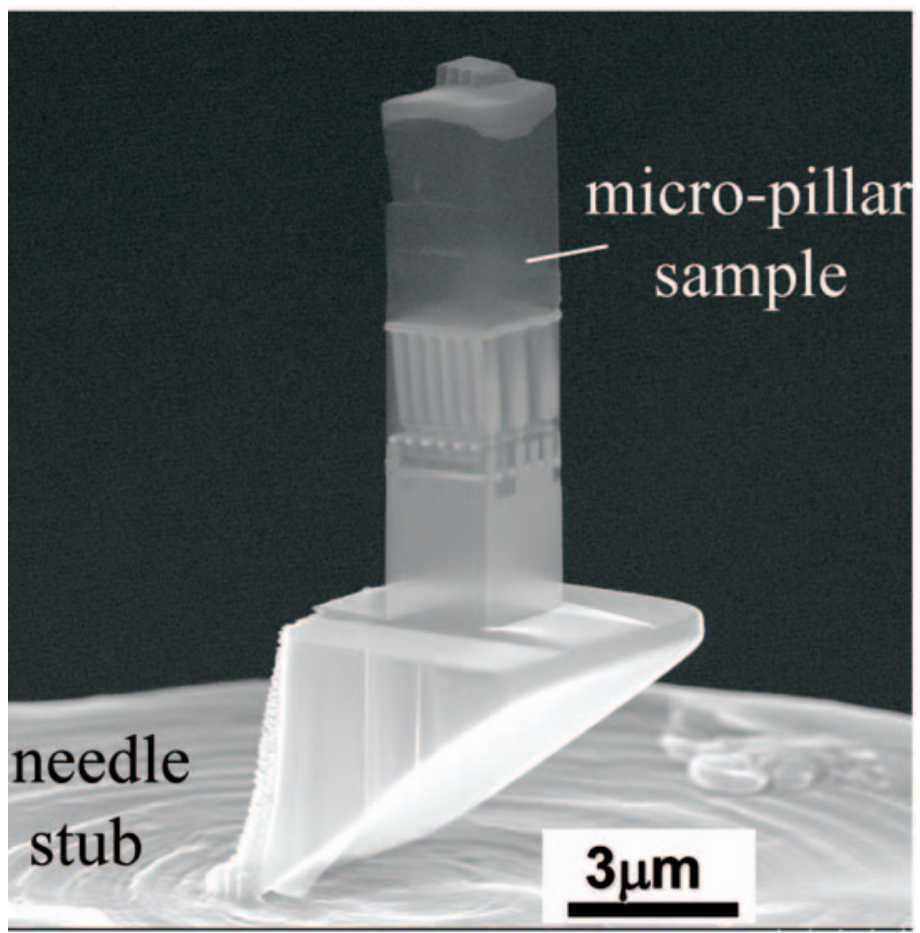

Figure 6. SE image of a micro-pillar specimen from a Si device mounted on a needle stub.

be located for further FIB milling, if required. Figure 7 shows a portion of a hemi-spherical grain (HSG) capacitor observed by SE (a), high angle annular dark-field (HAADF) STEM (b) and bright-field (BF) STEM (c) images. The shape and fine structure of the capacitor are observed three dimensionally.

Figure 8 shows high resolution STEM images of atom columns in a pillar-shaped $\mathrm{Si}$ single crystal specimen with a size of about $150 \mathrm{~nm} \times 150 \mathrm{~nm}$. Figure 8 (a) and (b) were observed using the [110] and the [1-10] diffraction directions respectively. A crystal lattice image of the $\mathrm{Si}$ (111) plane with the distance of $0.314 \mathrm{~nm}$ is observed clearly.

We have described a method for three-dimensional characterization of a specific-site defect, using the Hitachi FB-2100 FIB system equipped with a micro-sampling attachment and the Hitachi HD-2300 dedicated FE STEM. This method was applied for the structural characterization of SRAM and DRAM cells. The results revealed that the micro-sampling technique enabled
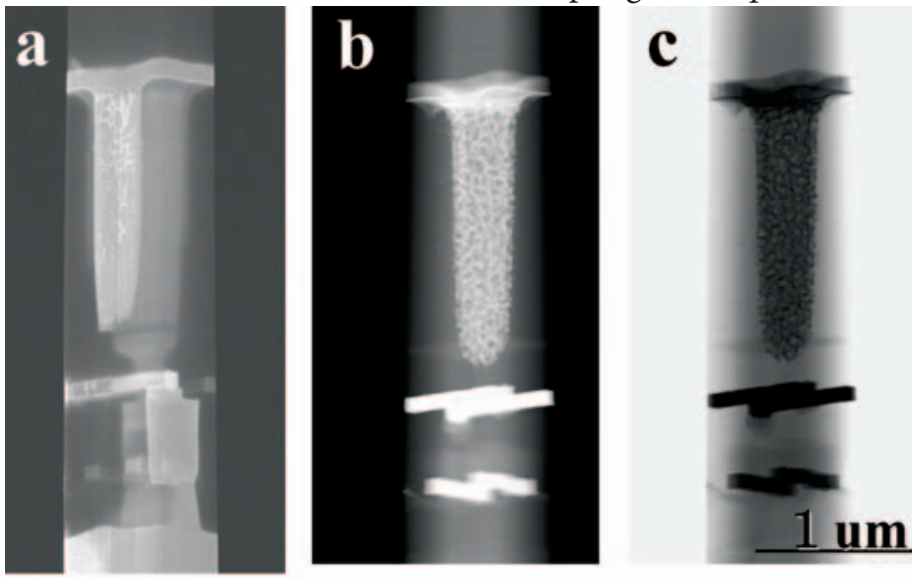

Figure 7. SE (a),DF-STEM (b), and BF-STEM (c) images of a 1 micron wide by 0.5 micron long square pillar. 


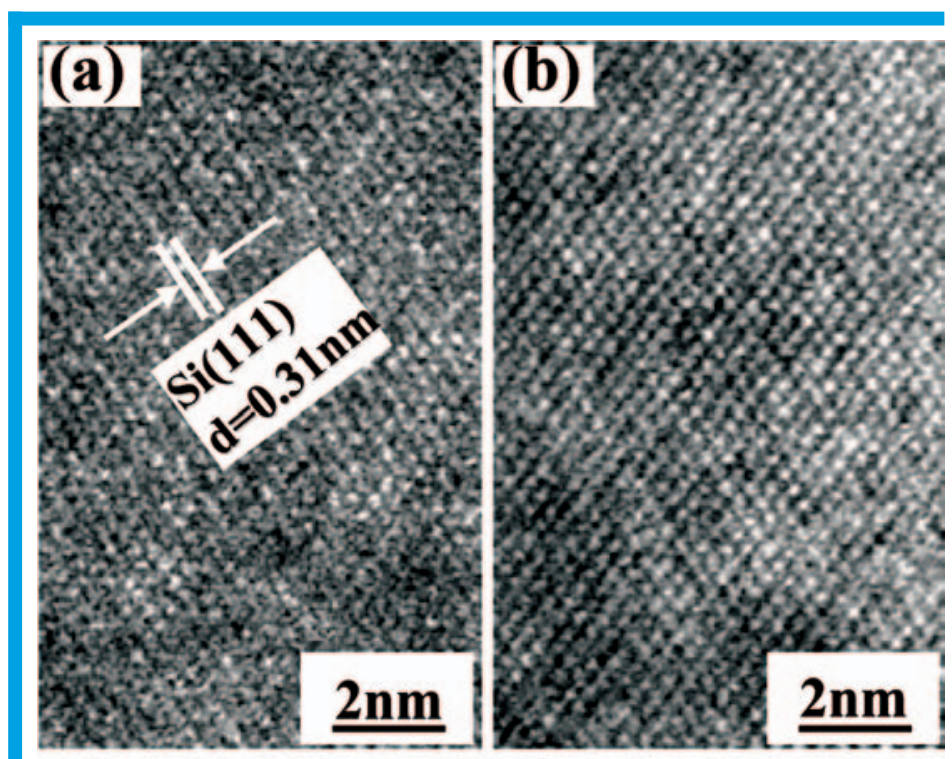

Figure 8. High resolution STEM images of a pillar-shaped Si single crystal specimen of about $150 \mathrm{~nm} \times 150 \mathrm{~nm}$. a.) [110] operating reflection,

b.) [1-10] operating reflection.

the observation of the three-dimensional structure of specific sites at atomic resolution and the technique can be applied to the failure analysis or evaluation of a wide range of materials.

\section{References}

1) T. Yaguchi, M. Konno, T. Kamino, T. Hashimoto, T. Ohnishi, K. Umemura and

K. Asayama, Proc. Microsc. Microanal. 9 (Suppl .2) (2003) 118-119.

2) T. Yaguchi, M. Konno, T. Kamino, T. Hashimoto, T.Ohnishi and K.Umemura

Proc. Microsc. Microanal. 10 (Suppl .2) (2004) 1164-1165.

3) T. Ohnishi and T. Ishitani,US patent 5270552

4) Tomimatsu et al. US patent 6538254.

SCDPETRENIX

PHONE/FAX

\section{Introducing the MaxView Plus} Digital Camera Attachment System

The MaxView Plus kit includes adapters to fit microscopes \& optical devices with any of the following attachments; C-Mount, C/S-Mount, T-Mount, 23mm Eyepiece Port, $30 \mathrm{~mm}$ Eyepiece Port, and 1.25" Eyepiece Ports

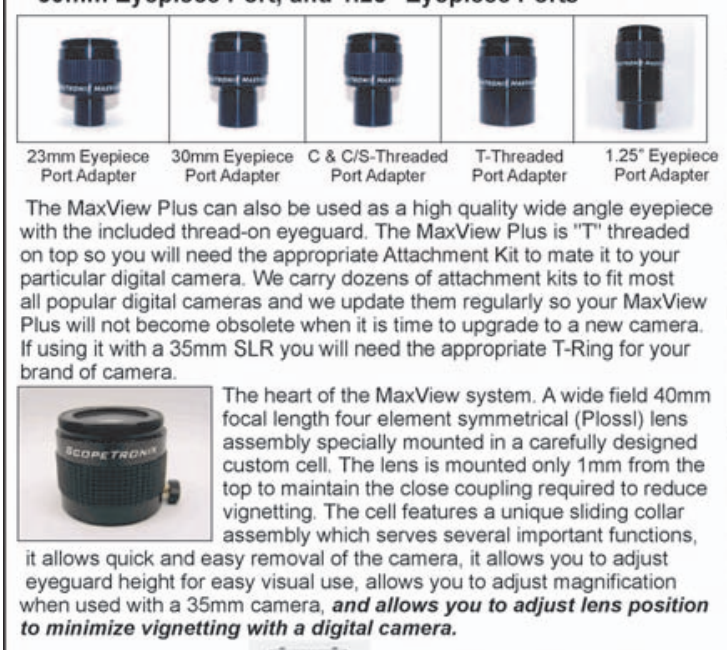
particular digital camera. We carry dozens of attachment kits to fit most all popular digital cameras and we update them regularly so your MaxView If using it with a $35 \mathrm{~mm}$ SLR you will need the appropriate T-Ring for your brand of camera.

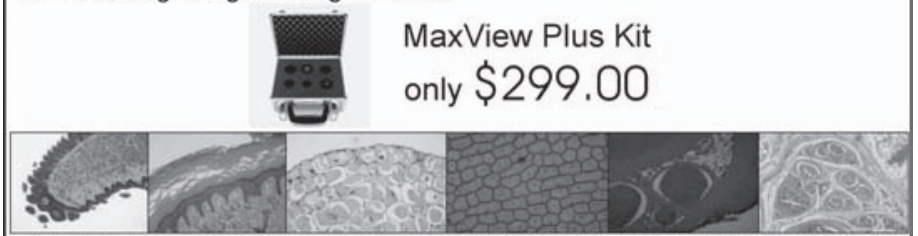

ScopeTronix Astronomy Products • 1423 SE 10th Street Unit 1A • Cape Coral, Florida 33990 Phone/FaX 24 HOUR GNLINE SHOPPING \& SECLRE ORDERIN (239) 945-6763 WMSCOPETRONX.COM
Expertise and joie de vivre... at McGill University in Montréal, Canada

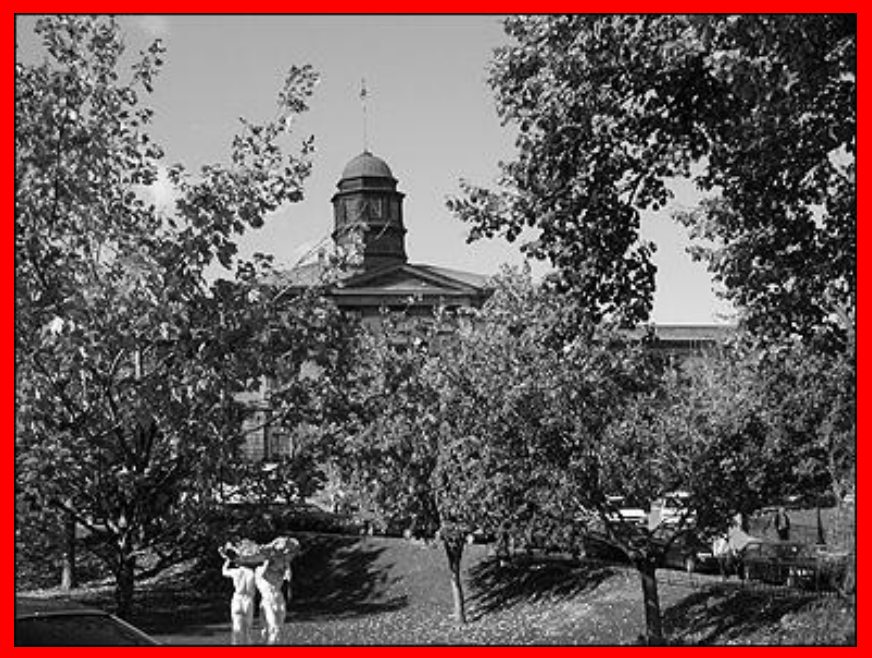

\section{"Advanced Techniques in} SEM and Microanalysis for Materials

\section{Characterization"}

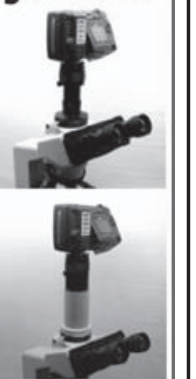

\section{Lecturers : David Joy Eric Lifshin Raynald Gauvin Pierre Hovington Marin Lagacé}

9 -13 May, 2005

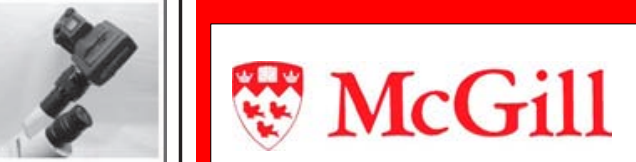

Department of Mining,

Metals an Materials

Engineering

McGill

Regroupement Aluminium

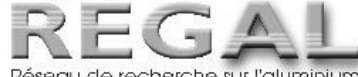
REGAL-McGill
Information : Prof. Raynald Gauvin (514) 398-8951

raynald.gauvin@mcgill.ca 